\title{
BIOMARKERS FOR MONITORING GYNECOLOGIC MALIGNANCIES
}

\author{
Suela Bushi ${ }^{1 *}$, Artan Trebicka ${ }^{1}$ \\ ${ }^{1 *}$ Faculty of Natural Sciences, Tirana, Albania; \\ *Corresponding Author Suela Bushi, e-mail: bushisuela@yahoo.it;
}

Received April 2021; Accepted May 2021; Published June 2021;

DOI: https://doi.org/10.31407/ijees11.319

\begin{abstract}
Tumor markers are playing an increasingly important role in cancer detection and management. These laboratorybased tests are potentially useful in screening for early malignancy, aiding cancer diagnosis, determining prognosis, surveillance following curative surgery for cancer, up front predicting drug response or resistance, and monitoring therapy in advanced disease. Tumor marker assays, CA15-3, CA 125, and CEA are valuable biomarkers for the differential diagnosis of patients with breast and ovarian cancer providing their well-known profiles of sensitivity and specificity and thus demonstrated their reliability for routine laboratory diagnostics. The aim of the study is to evaluate the hematological, biochemical and tumor markers changes changes in gynecologic malignancies after chemotherapy. Significant decrease of CA 125, CA 125 and CEA was observed in all three types of Ca following the second cycle of chemotherapy $(\mathrm{p}<0.01$ ). The values of hemato-biochemical parameters and tumor markers are important in identifying the course of therapy.
\end{abstract}

Key words: cancer, hematobiochemical parameters, tumor markers 